\title{
MiR-500a-5p promotes glioblastoma cell proliferation, migration and invasion by targeting chromodomain helicase DNA binding protein 5
}

\author{
ZHIYONG LIU ${ }^{1}$, DANYING SU ${ }^{2}$, XIUYING $\mathrm{QI}^{3}$ and JING MA ${ }^{3}$ \\ ${ }^{1}$ Laboratory of Neurology, The Class of 2014 Outstanding Physician, Jiamusi University, Jiamusi, \\ Heilongjiang 154007; ${ }^{2}$ Department of Neurology, The Fourth Affiliated Hospital of Harbin Medical University; \\ ${ }^{3}$ Department of Anatomy, Harbin Medical University, Harbin, Heilongjiang 150081, P.R. China
}

Received February 4, 2018; Accepted June 27, 2018

DOI: $10.3892 / \mathrm{mmr} .2018 .9259$

\begin{abstract}
Glioblastoma is one of the most common malignant primary tumors and develops in brain. The molecular mechanism that regulates glioblastoma occurrence still remains unknown. MicroRNA (miR)-500a-5p has been reported to be involved in hepatocellular carcinoma and breast cancer. Whether miR-500a-5p regulates glioblastoma progression requires further investigation. In the present study, miR-500a-5p was highly expressed in malignant glioblastoma tissues and cell lines. Overexpression of miR-500a-5p promoted glioblastoma cell proliferation, migration and invasion in vitro. In addition, knockdown of miR-500a-5p accelerated cell apoptosis. Furthermore, miR-500a-5p inhibition significantly impaired tumor growth in vivo. The present study further explored the downstream mechanism. The luciferase reporter assay revealed that miR-500a-5p directly binds the 3'-untranslated region of chromodomain helicase DNA binding protein 5 (CHD5) mRNA. MiR-500a-5p markedly inhibited CHD5 expression in glioblastoma cells. Furthermore, CHD5 was downregulated in glioblastoma tissues, and the expression levels of miR-500a-5p and CHD5 were inversely correlated. In addition, knockdown of CHD5 restored the inhibition of cell proliferation and migration triggered by miR-500a-5p silence. Finally, it was demonstrated that miR-500a-5p can serve as a novel biomarker for the diagnosis and prognosis of glioblastoma patients. Taken together, the results of the present study indicated that miR-500a-5p may have promoted glioblastoma development and progression by targeting CHD5.
\end{abstract}

Correspondence to: Professor Jing Ma, Department of Anatomy, Harbin Medical University, 194 Xuefu Road, Harbin, Heilongjiang 150081, P.R. China

E-mail: jingma002@163.com

Key words: microRNA-500a-5p, proliferation, migration, chromodomain helicase DNA binding protein 5, glioblastoma

\section{Introduction}

Glioblastoma is one of the most common malignant primary tumors and develops in brain (1). Glioblastoma is highly aggressive and the survival rate of patients with glioblastoma was very low $(2,3)$. Every year, large amounts of patients died from glioblastoma $(4,5)$. Surgical resection, radiotherapy and chemotherapy are the main methods for glioblastoma therapy. In spite of some advances in tumor therapy, treatments for glioblastomas still remain challenging. The tumor recurrence rate is still very high because of infiltrative growth of glioblastoma $(6,7)$. Up to now, many efforts have been made to understand the knowledge of glioblastoma biology. To develop gene therapies toward targeted sites, which show an exciting prospect, it is necessary to uncover the underlying molecular mechanisms that regulate glioblastoma.

MicroRNAs (miRNAs) belong to noncoding RNAs, which exert all kind of biological functions (8-10). miRNAs can bind to the 3'UTR of target mRNAs to promote their degradation and regulate gene expression with a post-transcriptional manner (11). Increasing evidences show that miRNAs participate in various cellular processes such as development, proliferation and tumor metastasis (12-15). Therefore, miRNAs may serve as effective therapeutic targets and diagnostic biomarkers. Previous study also showed that miRNAs possess important roles in glioblastomas. For example, MicroRNA-141-3p promotes glioblastoma cell growth by directly targeting p53 (16). However, most important miRNAs in glioblastoma remains to be revealed. As for miR-500a-5p, Zhao et al (17) reported that miR-500a overexpression enhances hepatocarcinoma metastasis by repressing PTEN expression. Degli et al (18), showed that miR-500a-5p regulates breast cancer progression and predicts cancer survival. Additionally, Guo et al (19), indicated that miR-500a promotes migration and invasion in hepatocellular carcinoma by activating the Wnt/ $\beta$-catenin signaling pathway. The role of miR-500a-5p in glioblastoma remains elusive and required to be defined.

In the present study, we aimed to investigate the function and mechanism of miR-500a-5p in glioblastoma. By reverse transcription-quantitative polymerase chain reaction (RT-qPCR), we showed that miR-500a-5p was upregulated in 
glioblastoma tissues and cells. CCK8 and Transwell assays indicated that miR-500a-5p overexpression promoted glioblastoma cell proliferation, migration and invasion in vitro. Moreover, xenograft experiments illustrated that miR-500a-5p inhibition delayed tumor growth in vivo. In mechanism, we found that miR-500a-5p directly targeted CHD5. Summarily, our study highlighted the essential role of miR-500a-5p on glioblastoma development and progression via miR-500a-5p/CHD5 axis.

\section{Materials and methods}

Patient samples. All 60 tissue samples were collected from Harbin Medical University (Heilongjiang, China). The present study received ethical approval from Harbin Medical University. The clinical characters of these samples were listed in Table I. Written informed consent approving this study was obtained from each patient. Human glioblastoma cell lines U-87MG (www.atcc.org/Products/All/HTB-14. aspx) and U251 were from the American Tissue Culture Collection (ATCC, Manassas, VA, USA). The U-87MG cell line is known to be cross-contaminated with another cell line of unknown origin, but it is likely to be another glioblastoma cell line (20). U-87MG cells were grown in Minimum Essential Medium (HyClone; GE Healthcare Life Sciences, Logan, UT, USA) with $10 \%$ fetal bovine serum (FBS; Gibco; Thermo Fisher Scientific, Inc., Waltham, MA, USA). U251 cells were maintained in Dulbecco's High Glucose Modified Eagle Medium (HyClone; GE Healthcare Life Sciences) with $10 \%$ FBS. Normal human astrocytes (NHA) obtained from Lonza (www.lonza.com/products-services/bio-research/ primary-cells/human-cells-and-media/neural-cells-and-media/ nha-normal-human-astrocytes.aspx) were cultured in the provided astrocyte growth media and 5\% FBS. Cells were incubated in a humidified atmosphere with $5 \% \mathrm{CO}_{2}$ at $37^{\circ} \mathrm{C}$.

Construction and infection. NC mimic, miR-500a-5p mimic and its corresponding miR-500a-5p inhibitor were purchased from Guangzhou RiboBio Co., Ltd., (Guangzhou, China). Cell transfection was performed with Lipofectamine 2000 Reagent (Invitrogen; Thermo Fisher Scientific, Inc.) according to manufacturer's protocol. After incubating, cells were harvested then experiments were performed. Loss of CHD5 expression was achieved using small interfering RNA (siRNA) of CHD5. To establish a stably CHD5-silenced cell line, a GV113 plasmid containing CHD5 lentivirus short hairpin RNAs (TGGTTA AGGGCAGTGATAG) were separately transduced into U-87MG cells.

CCK8 assay. Cell proliferation was detected by Cell Counting Kit (7 sea biotech, Shanghai, China). Cells were grown in 96-well plate with $1 \times 10^{4}$ per well and incubated in $37^{\circ} \mathrm{C}$ with $5 \% \mathrm{CO}_{2}$ until cell confluent rate reached $70 \%$. After transfected with plasmid for $48 \mathrm{~h}$, cells were still incubated for 24,48 and 72 h. $10 \mu 1$ CCK 8 solution was seed into each well. The absorbance at $450 \mathrm{~nm}$ was measured with SUNRISE Microplate Reader (Tecan Group, Ltd., Mannedorf, Switzerland).

Apoptosis assay. Cell apoptosis was measured by flow cytometry following the instructions of the Annexin V-FITC/ PI apoptosis detection kit (Nanjing KeyGen Biotech Co., Ltd.,
Table I. Clinical parameters of microRNA-500a-5p in glioblastoma samples.

\begin{tabular}{lccc}
\hline Characteristics & Low expression & High expression & P-value \\
\hline $\begin{array}{l}\text { Age (years) } \\
\leq 60\end{array}$ & 19 & 18 & 1.00 \\
$>60$ & 11 & 12 & \\
Gender & & & 0.43 \\
Male & 20 & 16 & \\
Female & 10 & 14 & \\
Grade & & & 0.02 \\
II & 14 & 6 & \\
III & 10 & 8 & \\
IV & 6 & 16 & \\
Tumor size (cm) & & & 0.01 \\
$\leq 4$ & 17 & 6 & \\
$>4$ & 13 & 24 & \\
\hline
\end{tabular}

Nanjing, China). Cultured cells were harvested and washed twice in PBS, re-suspended in binding buffer, and then incubated with Annexin V-FITC and propidium iodide (PI) for $15 \mathrm{~min}$ at room temperature. Afterwards, flow cytometry was performed to determine rate of apoptosis on a FACSAria flow cytometer (BD Biosciences, Franklin Lakes, NJ, USA).

In vitro migration and invasion assays. Twenty-four-well Transwell chambers with $8-\mu \mathrm{m}$ pore size polycarbonate (Corning Incorporated, Corning, NY, USA) were used for cell migration and invasion assays. For invasion assays, the top side of the membrane was coated with Matrigel (BD Biosciences), and then $1 \times 10^{5}$ cells (in each well) in serum-free DMEM or RPMI 1640 medium were seeded on the chambers. DMEM or RPMI 1640 containing 10\% FBS was added to the wells under the chamber. For migration analysis, $5 \times 10^{4}$ cells (in each well) in serum-free DMEM or RPMI 1640 medium were seeded on the chambers without Matrigel. After $24 \mathrm{~h}$ of incubation, cotton swabs were used to remove the cells inside the upper chamber, while the cells on the other side of the membrane surface were fixed and stained with $0.5 \%$ crystal violet solution. Five random fields were counted in each well.

Bioinformatics analysis. The potential targets of miR-500a-5p were predicted using the TargetScan (www.targetscan.org), microRNA (www.microrna.org/) and miRDB (www.mirdb. org). The common genes of these algorithms were selected for further analysis.

$R T-q P C R$. The total RNA of the tissue samples and cells was isolated using TRIzol Reagent (Invitrogen; Thermo Fisher Scientific, Inc.), in accordance with manufacturer's instructions. Then, $1 \mu \mathrm{g}$ of total RNA was reverse-transcribed in a volume of $20 \mu \mathrm{l}$ using random and oligo dT primers under standard conditions, in accordance with the instructions of the PrimeScript RT kit (Takara Biotechnology Co., Ltd., Dalian, China). For RT-qPCR assays, we used SYBR Premix Ex Taq (Takara Biotechnology Co., Ltd.) to determine the expression 
level of CHD5, in accordance with manufacturer's instructions. The thermocycling conditions were as follows: $95^{\circ} \mathrm{C}$ for $30 \mathrm{sec}$, followed by 40 cycles at $95^{\circ} \mathrm{C}$ for $15 \mathrm{sec}$ and $60^{\circ} \mathrm{C}$ for $32 \mathrm{sec}$ and dissociation at $95^{\circ} \mathrm{C}$ for $60 \mathrm{sec}, 55^{\circ} \mathrm{C}$ for $30 \mathrm{sec}$ and $95^{\circ} \mathrm{C}$ for $30 \mathrm{sec}$. The expression data of CHD5 were normalized to the expression of glyceraldehyde-3-phosphate dehydrogenase (GAPDH). The primers used were as follows: Forward primer, 5'-CTGACTTCAACAGCGACACC-3' and reverse primer, 5'-TCTGACTTCAACAGCGACACC-3'. The relative fold change was calculated using the $2^{-\Delta \Delta \mathrm{Cq}}$ method (21).

In vivo assay. The protocol was described previously (22). Four-week-old athymic BALB/c nude mice were maintained under specific pathogen-free conditions. The mice were manipulated in accordance with the protocols approved by Harbin Medical University. The U-87MG cells were harvested and washed with PBS. Then, $1 \times 10^{7}$ cells were subcutaneously injected into the ventral side of each mouse for tumor formation assays. Six mice were used for each group. The tumor volumes were examined every 7 days and calculated. The protocol was approved by the Animal Care Ethics Committee of Harbin Medical University.

Details in Sukru's cohort (GSE90598). In this dataset, 16 fresh-frozen glioblastoma multiforme samples, 7 healthy brain tissues, a NHA cell line and human fetal astrocyte cell line were analyzed by using miRNA and whole transcriptome microarray chips.

Luciferase reporter assay. U-87MG cells were seeded into a 24-well plate. Cells were co-transfected with wild-type, mutated CHD5 reporter plasmid or pMIR vector, and miR-500a-5p mimics or miR-500a-5p inhibitor. Luciferase assays were conducted $24 \mathrm{~h}$ after transfection using the Dual Luciferase Reporter Assay System (Promega Corporation, Madison, WI, USA).

Statistical snalysis. Statistical analysis was performed using SPSS v18.0 software (SPSS, Inc., Chicago, IL, USA). Each experiment was repeated at least three times. All data are expressed as the mean \pm standard deviation. The Kaplan-Meier method was used to calculate the survival curve, and log-rank test to determine statistical significance. Student's t-test and one-way analysis of variance followed by Tukey's post hoc test were used to analyze 2 or multiple groups, respectively, for statistical significance. A paired Student's t-test was used to compare differences between human glioblastoma and normal adjacent tissue samples. Diagnostic potential of miR-500a-5p expression was assessed by receiver operating characteristic (ROC) analysis. The respective area under the curve (AUC) was analyzed by the Hanley and McNeil method. For analysis of correlation between miR-500a-5p levels and clinical features, chi-square tests were used. Pearson correlation coefficient analysis was used to determine the correlations. $\mathrm{P}<0.05$ was considered to indicate a statistically significant difference.

\section{Results}

MiR-500a-5p is significantly upregulated in glioblastoma tissues. To explore the function miR-500a-5p, we firstly examined its expression in glioblastoma tissues by RT-qPCR. We found that miR-500a-5p was significantly upregulated in glioblastoma tissues compared with adjacent normal tissues (Fig. 1A). Similarly, the high expression of miR-500a-5p was verified in glioblastoma cell lines (Fig. 1B). Then we divided these samples into two groups according to miR-500a-5p expression levels (mean value was the cut-off). Kaplan-Meier analysis was used to evaluate survival. The results showed that patients with higher miR-500a-5p expression possessed poorer survival (Fig. 1C). Furthermore, ROC curves were performed to evaluate the sensitivity and specificity of miR-500a-5p expression in predicting glioblastoma tissues from normal tissues. Notably, miR-500a-5p displayed predictive, with an AUC of 0.753 ( $\mathrm{P}=0.046$; Fig. 1D). These results implied that miR-500a-5p might provide imperative clinical significance in glioblastoma diagnosis.

MiR-500a-5p enhances glioblastoma cell proliferation, migration and invasion in vitro. To determine the functions of miR-500a-5p in glioblastoma, we overexpressed miR-500a-5p in $\mathrm{U}-87 \mathrm{MG}$ and $\mathrm{U} 251$ cells by transduction with $\mathrm{NC}$ mimic or miR-500a-5p mimic. At $48 \mathrm{~h}$ after transduction with miR-500a-5p, miR-500a-5p expression was significantly increased compared with NC mimic group (Fig. 2A). Then CCK8 assay was conducted to analyze the proliferation of $\mathrm{U}-87 \mathrm{MG}$ and U251 cells at 24, 48 and $72 \mathrm{~h}$ post transfection (Fig. 2B). Overexpression of miR-500a-5p observably promoted cell proliferation in both U-87MG MG and U251 cells. Similarly, colony formation assay showed that cells transfected with miR-500a-5p formed more clones (Fig. 2C). In consistence, we found that more U-87MG and U251 cells transfected with miR-500a-5p entered into $S$ phase than control (Fig. 2D). To evaluate the effect of miR-500a-5p on cell migration and invasion, we performed transwell assays. We found that overexpression of miR-500a-5p promoted cell migration and invasion (Fig. $2 \mathrm{E}$ and $\mathrm{F}$ ). In addition, we inhibited miR-500a-5p in U-87MG and U251 cells to evaluate the effect on cell apoptosis. Flow Cytometry by Annexin V/PI staining showed that apoptotic ratio was significantly higher in miR-500a-5p inhibitor-transfected cells than control (Fig. 2G). In collection, miR-500a-5p promoted cell proliferation, migration and invasion in glioblastoma, but inhibited cell apoptosis.

MiR-500a-5p delayed tumor growth in vivo. To further determine the effects of miR-500a-5p on glioblastoma cells in vivo, we examined tumor growth in nude mice. U-87MG cells transfected with $\mathrm{NC}$ inhibitor or miR-500a-5p inhibitor were subcutaneously inoculated into the armpits of the recipient nude mice. Every other 7 day, we measured the tumor volumes. We found that miR-500a-5p knockdown significantly inhibited tumor growth in vivo (Fig. 3A). At the endpoint of the experiments, the tumor weights were measured. The tumors in miR-500a-5p knockdown group were significantly lighter than that in control group (Fig. 3B).

MiR-500a-5p specifically targeted CHD5 3'-UTR in glioblastoma. To explore the molecular mechanism through which miR-500a-5p promoted glioblastoma progression, three computational algorithms including TargetScan, miRanda and PicTar were used in combination to search for potential targets of 
A

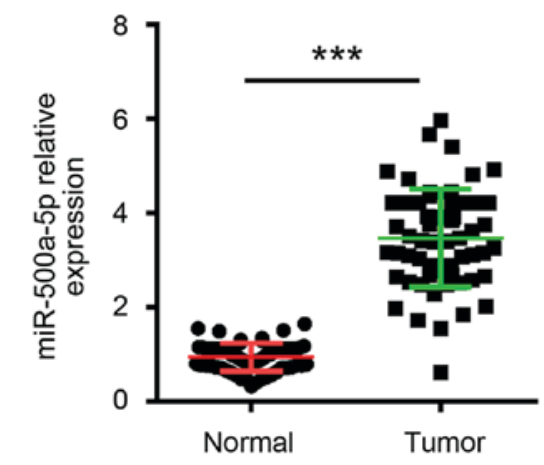

C

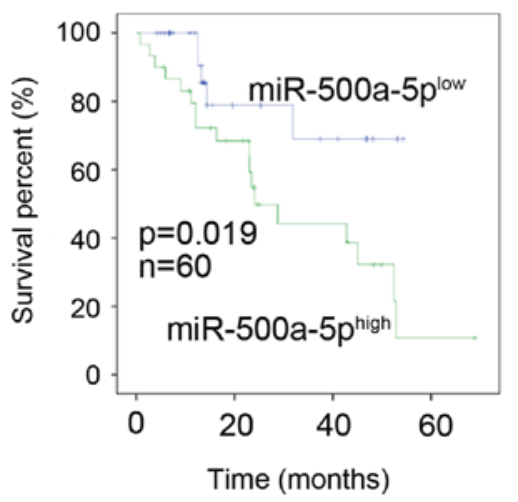

B

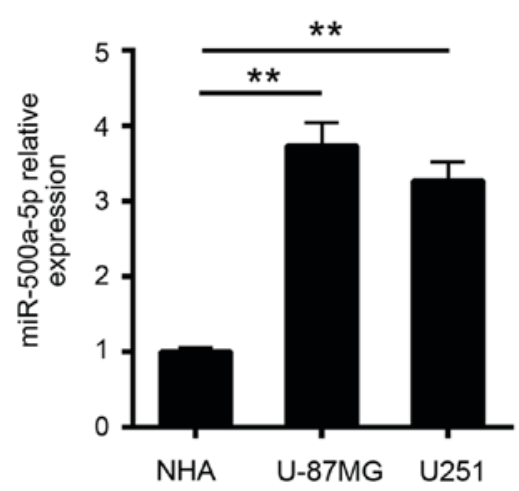

D

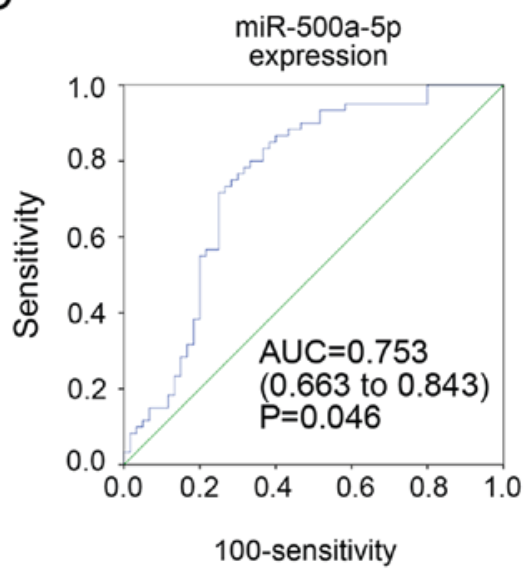

Figure 1. miR-500a-5p is significantly upregulated in glioblastoma tissues. (A) RT-qPCR assay of miR-500a-5p expression in glioblastoma and normal tissues (B) RT-qPCR assay of the miR-500a-5p levels in glioblastoma cells and NHAs. (C) Kaplan-Meier survival curve according to miR-500a-5p expression levels. (D) ROC analysis based on miR-500a-5p expression for survival prediction of patients with glioblastoma. All data are representative of three independent experiments and expressed as the mean \pm standard deviation. ${ }^{* *} \mathrm{P}<0.01$ and ${ }^{* * *} \mathrm{P}<0.001$, as indicated. miR, microRNA; RT-qPCR, reverse transcription-quantitative polymerase chain reaction; NHA, normal human astrocytes; AUC, area under the curve; ROC, receiver operating characteristic.

miR-500a-5p. Among the candidates, the chromatin remodeler and tumor suppressor CHD5 was predicted to be a target of miR-500a-5p. The predicted interaction between miR-500a-5p and 3'UTR of CHD5 was illustrated (Fig. 4A). In order to verify this prediction, we cloned 3'UTR-wt and 3'UTR-mut into pMIR-REPORT vector. As expected, dual luciferase assay demonstrated that miR-500a-5p overexpression remarkably inhibited the luciferase activity while miR-500a-5p knockdown increased the luciferase activity (Fig. 4B). In contrast, the effect of miR-500a-5p on luciferase activity observed in pMIR-3'-UTR-wt was absent in pMIR-3'-UTR-mut (Fig. 4B). Moreover, we found that overexpression of miR-500a-5p decreased the mRNA and protein levels of CHD5 in U-87MG and U251 cells while miR-500a-5p knockdown got the inverse result (Fig. 4C and D). In addition, by RT-qPCR we found that the expression of miR-500a-5p was inversely correlated with that of CHD5 in glioblastoma tissues (Fig. 4E). Furthermore, the dataset (GSE90598) in GEO database (https://www.ncbi.nlm.nih.gov/geo/query/acc. cgi?acc=GSE90598) showed that the expression of CHD5 was significantly downregulated in glioblastoma tissues compared with normal tissues (Fig. 4F). Similarly, RT-qPCR also showed that the mRNA levels of CHD5 were lower in glioblastoma samples than normal tissues (Fig. 4G). These results indicated that CHD5 mRNA 3'-UTR is a specific functional target of miR-500a-5p in glioblastoma cells.
MiR-500a-5p regulated glioblastoma cell proliferation, migration and invasion by targeting CHD5 in vitro and in vivo. To investigate whether the regulation of cell proliferation, migration and invasion of glioblastoma cells by miR-500a-5p is executed via a CHD5-dependent manner, we co-transfected U-87MG and U251 cells with miR-500a-5p inhibitor and CHD5 siRNA. Compared with cells transfected with miR-500a-5p inhibitor, the cells transfected with both miR-500a-5p inhibitor and CHD5 siRNA exhibited a lower expression on CHD5 protein level (Fig. 5A). CCK8 and colony formation assays showed that CHD5 knockdown reversed the inhibitory effects by miR-500a-5p inhibition on cell proliferation potential in U-87MG and U251 cells (Fig. 5B and C). In addition, transwell assays showed that CHD5 knockdown reversed the inhibitory effects by miR-500a-5p inhibition on cell migration and invasion potential in U-87MG and U251 cells (Fig. 5D and E). Furthermore, CHD5 knockdown decreased the cell apoptosis induced by miR-500a-5p inhibition (Fig. 5F). What's more, CHD5 knockdown also increased tumor weights to the control level in vivo (Fig. 5G). Besides, WB assay with formed tumor tissues showed that CHD5 knockdown reversed the inhibitory effects by miR-500a-5p inhibition on cell proliferation, migration and invasion in vivo (Fig. $5 \mathrm{H}$ ). Accumulating studies showed that Wnt/ $\beta$-catenin signaling is indispensable for cancer development. We then performed WB assays with formed 
A

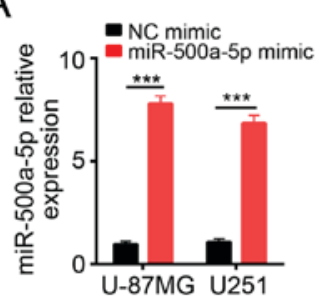

B

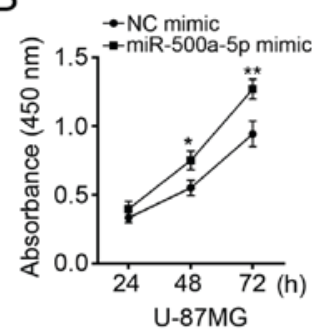

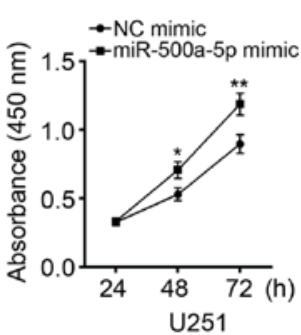

U251
C

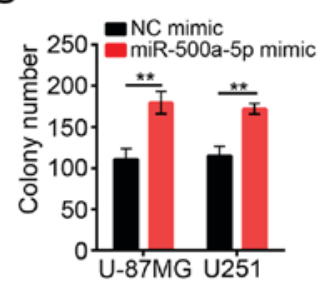

D
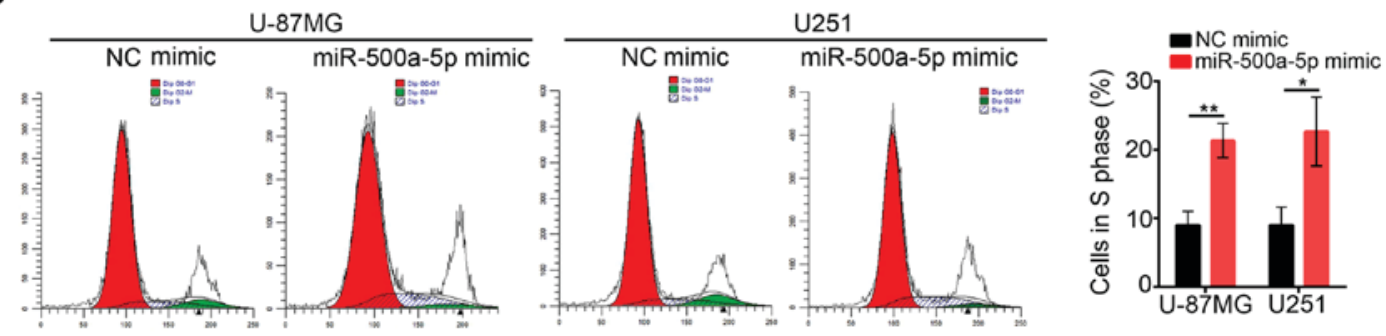

E
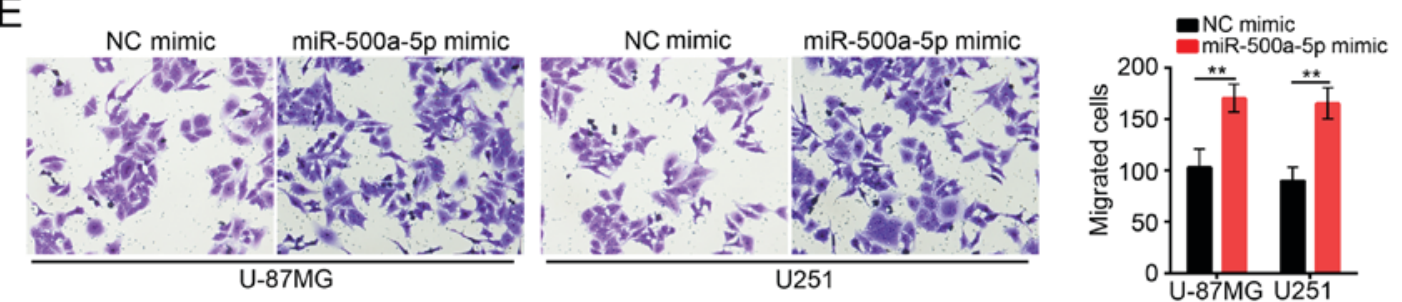

F
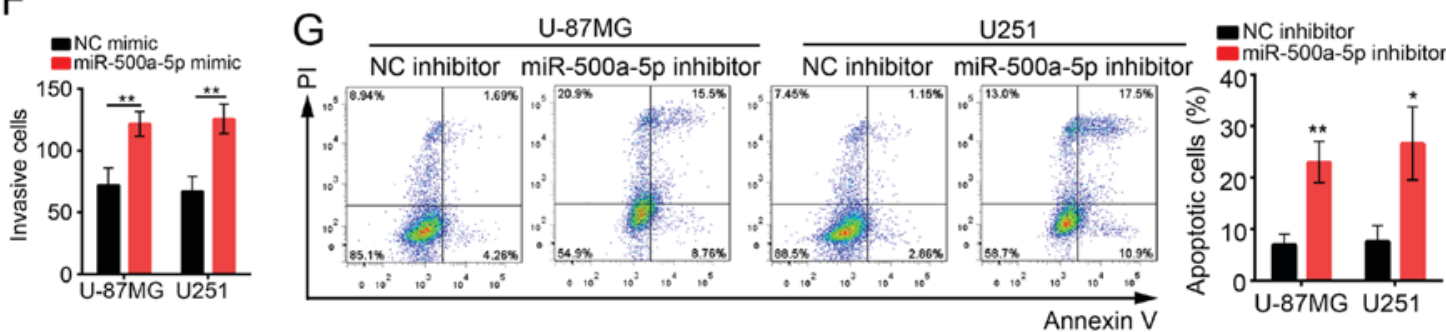

Figure 2. miR-500a-5p enhances glioblastoma cell proliferation, migration and invasion in vitro and in vivo. (A) Reverse transcription-quantitative polymerase chain reaction assay of the miR-500a-5p level in U-87MG and U251 cells transfected with NC mimic or miR-500a-5p mimic. (B) Cell Counting Kit-8 proliferation assay revealed the effects of miR-500a-5p overexpression on U-87MG and U251 cell proliferation. (C) Colony formation assay demonstrated the effects of miR-500a-5p overexpression on U-87MG and U251 cell proliferation. (D) FACS assay revealed that miR-500a-5p overexpression increased the number of cells in the S phase. (E) Migration and (F) invasion assays demonstrated that miR-500a-5p overexpression promoted the migration and invasion of U-87MG and U251 cells (magnification, x200). (G) FACS assay by staining with Annexin V/PI showed that knockdown of miR-500a-5p promoted U-87MG and U251 cell apoptosis. All data are representative of three independent experiments and expressed as the mean \pm standard deviation. ${ }^{*} \mathrm{P}<0.05,{ }^{* *} \mathrm{P}<0.01$ and ${ }^{* * * *} \mathrm{P}<0.001$ vs. the NC group. FACS, fluorescence activated cell sorter; miR, microRNA; PI, propidium; NC, negative control.

tumor tissues and found that miR-500a-5p inhibition downregulated Wnt/ $\beta$-catenin signaling while CHD5 knockdown upregulated it (Fig. 5I), which indicated that miR-500a-5p/ CHD5 regulated glioblastoma development and progression through $\mathrm{Wnt} / \beta$-catenin signaling at least in part.

\section{Discussion}

Glioblastoma was one of the most common clinical primary brain tumors. However, the mechanism that regulates glioblastoma development and progression remains largely unknown. Previous studies demonstrated that miRNAs exerted pivot functions in all kinds of cancers including pancreatic cancer, glioblastoma, breast carcinoma, hepatocellular carcinoma and so on (23-26). In glioblastoma, some
miRNAs are reported to exert important functions. For instance, microRNA-101 inhibits proliferation, migration and invasion of human glioblastoma by targeting SOX9 (27). Nevertheless, the functions of most of miRNAs are unknown in glioblastoma. Therefore, there is an urgent need to define the molecular mechanism that regulates the genesis of glioblastoma, in order to develop effective therapeutics. Previous research shows that miR-500a increases cancer stem cells properties by STAT3 pathway in human hepatocellular carcinoma (28). Besides, MicroRNA-500a also enhances migration and invasion in hepatocellular carcinoma by activating the Wnt/ $\beta$-catenin signaling pathway (19). However, the functions of miR-500a-5p remain to be elucidated in glioblastoma. In our study, we showed that the expression of miR-500a-5p was significantly upregulated in glioblastoma tissues compared to 
A

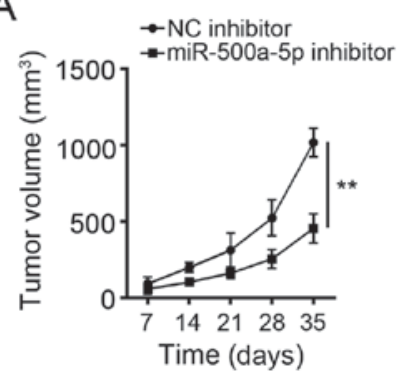

B

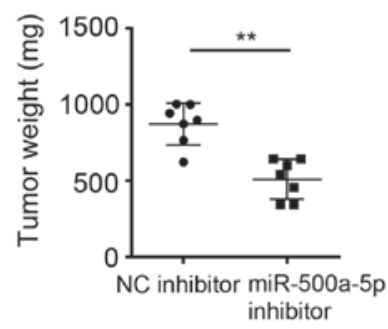

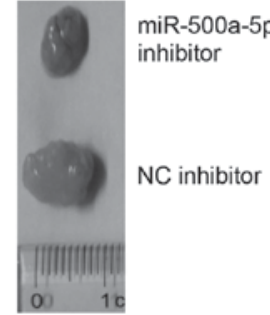

Figure 3. miR-500a-5p delays tumor growth in vivo. (A) Growth curve of U-87MG xenograft tumors; volume was measured every seven days. (B) Tumor weights were analyzed at the end of experiments. All data are representative of three independent experiments and expressed as the mean \pm standard deviation. ${ }^{* *} \mathrm{P}<0.01$, as indicated. miR, microRNA; NC, negative control.

A

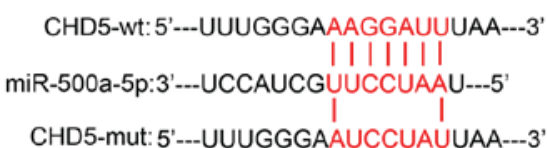

D

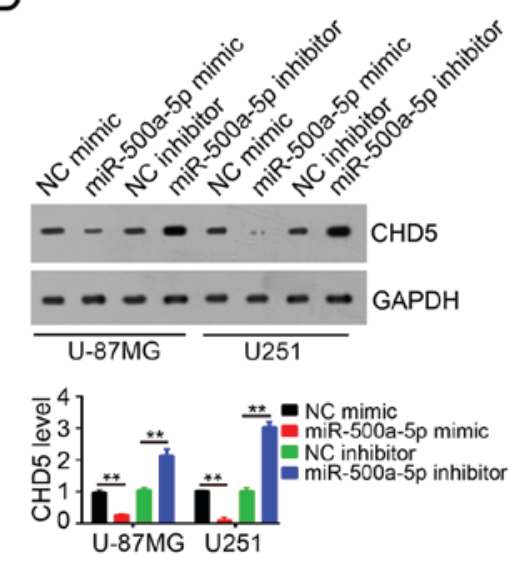

B

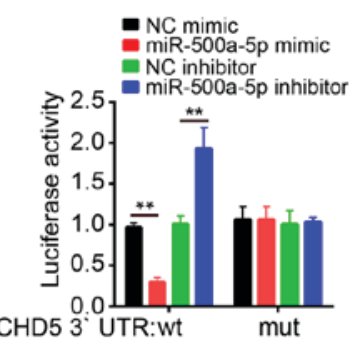

C

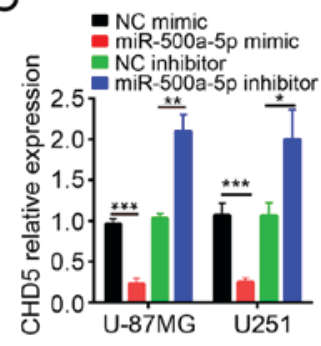

$\mathrm{E}$

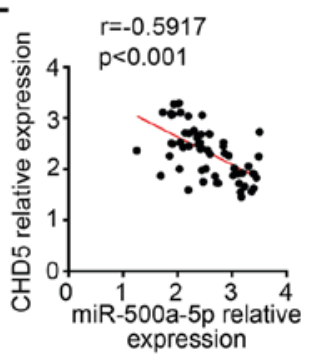

$\mathrm{F}$

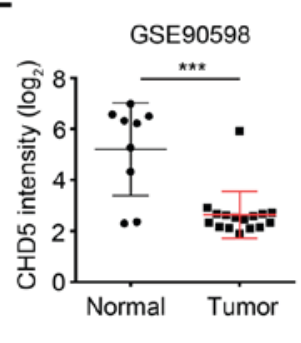

G

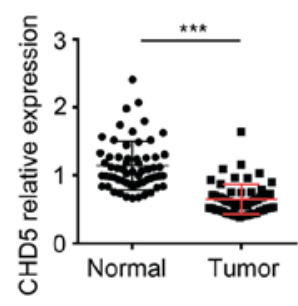

Figure 4. miR-500a-5p specifically targets CHD5 3'-untranslated region in glioblastoma. (A) A diagram presenting the binding site of miR-500a-5p in CHD5. (B) Dual-luciferase reporter assay revealed that co-transfection with miR-500a-5p significantly reduces luciferase activity of the reporter containing the CHD5-wt, but it has less of an effect on the reporter containing CHD5-mut in U-87MG cells. (C) RT-qPCR and (D) western blotting assays demonstrated the effects of miR-500a-5p down- or upregulation on CHD5 expression in U-87MG and U251 cells. (E) There was an inverse correlation between miR-500a-5p and CHD5 expression in glioblastoma tissues. (F) CHD5 was downregulated in glioblastoma tissues according to the dataset (GSE90598). (G) RT-qPCR assay showed that CHD5 was downregulated in glioblastoma tissues when compared with normal tissues. All data are representative of three independent experiments and are expressed as the mean \pm standard deviation. ${ }^{*} \mathrm{P}<0.05,{ }^{* *} \mathrm{P}<0.01$ and ${ }^{* * *} \mathrm{P}<0.001$ vs. the control group. miR, microRNA; RT-qPCR, reverse transcription-quantitative polymerase chain reaction; CHD5, chromodomain helicase DNA binding protein 5; wt, wild type; mut, mutant; NC, negative control.

normal tissues, which indicated that miR-500a-5p may act as on oncogene in glioblastoma.

Aberrant miRNAs expression is closely related to various types of tumors (29). In the present study, we demonstrated the highly expression of miR-500a-5p in glioblastoma tissues and cell lines. In addition, by analysis with Kaplan-Meier survival curve and ROC, we showed that the expression of miR-500a-5p in glioblastoma can serve as a new biomarker for the diagnosis and prognosis of patients with glioblastoma. To further determine the roles of miR-500a-5p in glioblastoma, we overexpressed miR-500a-5p in U-87MG and U251 cells. By CCK8, colony formation and transwell assays, we showed that overexpression of miR-500a-5p promoted cell proliferation, migration and invasion in U-87MG and U251 cells in vitro. Besides, we also knocked down miR-500a-5p by transfection with miR-500a-5p inhibitor. By FACS with Annexin V/PI staining, we found that miR-500a-5p increased the apoptosis of U-87MG and U251 cells. To further explore the physiological function of miR-500a-5p, we conducted xenograft experiments, which indicated that miR-500a-5p inhibition significantly inhibited glioblastoma growth in vivo.

Up to now, the targets of miR-500a-5p have not been identified. On the basis of bioinformatics analysis, we predicted CHD5 as a target of miR-500a-5p in glioblastoma. CHD5 is a chromatin remodeler and serves as a tumor suppressor in various tumors. For example, CHD5 is a potential tumor suppressor in non-small cell lung cancer 
A
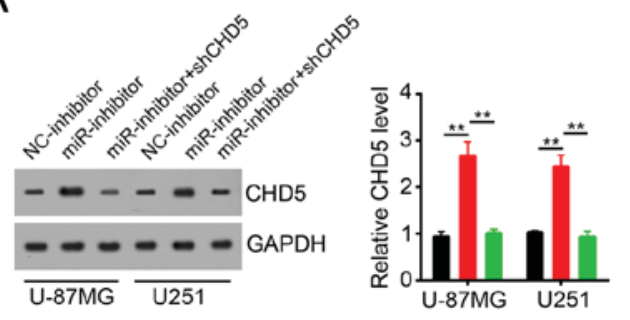

B

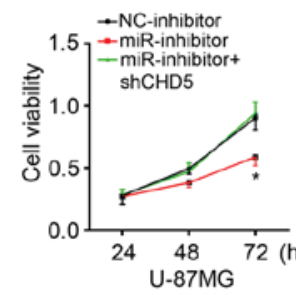

C

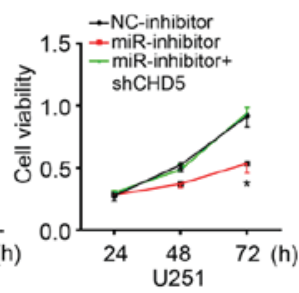

$\mathrm{F}$

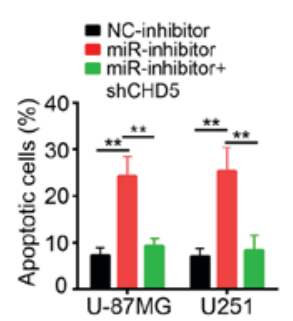

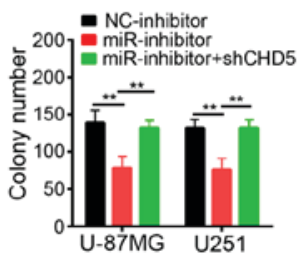

D

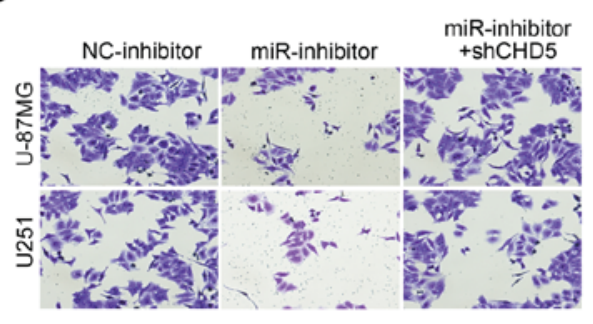

E

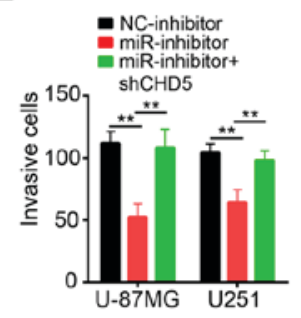

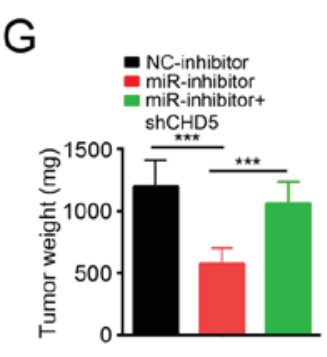

$\mathrm{H}$

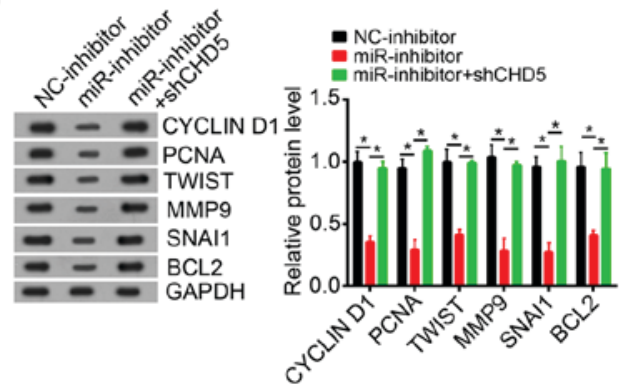

I

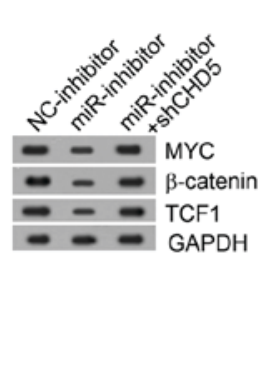

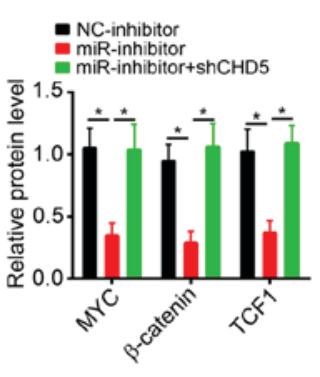

Figure 5. miR-500a-5p regulates glioblastoma cell proliferation, migration and invasion by targeting CHD5 in vitro and in vivo. (A) Western blot assay showed the protein levels of CHD5 in U-87MG and U251 cells transfected with miR-363-3p inhibitor. (B) Cell Counting Kit-8 proliferation assay demonstrated the effects of miR-500a-5p and/or CHD5 knockdown on U-87MG and U251 cell proliferation. (C) Colony formation assay revealed the effects of miR-500a-5p and/or CHD5 knockdown on U-87MG and U251 cell proliferation. (D and E) Migration and invasion assays demonstrated the effects of miR-500a-5p and/or CHD5 knockdown on the migration and invasion of U-87MG and U251 cells (magnification, x200). (F) Fluorescence activated cell sorter assay by staining with Annexin V/PI showed the effects of miR-500a-5p and/or CHD5 knockdown on U-87MG and U251 cell apoptosis. (G) Tumor weights of U-87MG xenograft tumors were measured at the end of experiments. (H) Western blot assay revealed the effects of miR-500a-5p and/or CHD5 knockdown on tumor proliferation and migration in vivo. (I) Western blot assay indicated that miR-500a-5p activated Wnt/ $\beta$-catenin signaling by targeting CHD5 in glioblastoma. All data are representative of three independent experiments and expressed as the mean \pm standard deviation. ${ }^{*} \mathrm{P}<0.05,{ }^{* * *} \mathrm{P}<0.01$ and ${ }^{* * * *} \mathrm{P}<0.001 \mathrm{vs}$. the $\mathrm{NC}$ group. miR, microRNA; CHD5, chromodomain helicase DNA binding protein 5; PI, propidium iodide; sh-, short hairpin RNA; TCF1, transcription factor 7; BCL2, B-cell lymphoma-2; PCNA, proliferating cell nuclear antigen; TWIST, Twist family basic helix-loop-helix transcription factor; MMP9, matrix metalloproteinase; SNAI1, Snail family transcription repressor 1; NC, negative control.

(NSCLC) (30). In glioblastoma, a report showed that CHD5 is downregulated in human glioblastoma (31). Nevertheless, how the expression of CHD5 is regulated remains unknown in glioblastoma. By luciferase reporter assay, we showed that the miR-500a-5p directly bond to the 3'-UTR of CHD5 mRNA. RT-qPCR and WB results showed that miR-500a-5p inhibited the mRNA and protein level of CHD5 in glioblastoma cells. Besides, we showed that miR-500a-5p activated Wnt/ $\beta$-catenin signaling while CHD5 inhibited this signaling in glioblastoma. Our results indicated that miR-500a-5p promoted cell proliferation, migration and invasion by targeting CHD5 and subsequently activating Wnt/ $\beta$-catenin signaling. However, how CHD5 mediates activation of $\mathrm{Wnt} / \beta$-catenin pathway needs further investigation. And the roles of CHD5-mediated activation of Wnt/ $\beta$-catenin signaling on glioblastoma progression still require to be defined.
In summary, the present study provides new insights into the mechanism of glioblastoma progression, and suggests that miR-500a-5p might potentially serve as therapeutic target for glioblastoma.

\section{Acknowledgements}

Not applicable.

\section{Funding}

No funding was received.

\section{Availability of data and materials}

All data generated or analyzed during this study are included in this published article. 


\section{Authors' contributions}

ZL and JM conceived and designed the present study, analyzed and interpreted the results, and wrote the manuscript. DS and XQ performed the experiments. All authors read and approved the final manuscript.

\section{Ethics approval and consent to participate}

For the use of human samples, the protocol for the present study was approved by the Institutional Ethics Committee of Harbin Medical University (Heilongjiang, China) and all enrolled patients signed a written informed consent document. In addition, all procedures involving animals conformed to the national guidelines of and were approved by the Animal Care Ethics Committee of Harbin Medical University.

\section{Patient consent for publication}

All patients recruited to the present study provided written informed consent for the publication of their data.

\section{Competing interests}

The authors declare that they have no competing interests.

\section{References}

1. Ilkanizadeh S, Lau J, Huang M, Foster DJ, Wong R, Frantz A, Wang S, Weiss WA and Persson AI: Glial progenitors as targets for transformation in glioma. Adv Cancer Res 121: 1-65, 2014.

2. Zhang K, Sun X, Zhou X, Han L, Chen L, Shi Z, Zhang A, Ye M, Wang Q, Liu C, et al: Long non-coding RNA HOTAIR promotes glioblastoma cell cycle progression in an EZH2 dependent manner. Oncotarget 6: 537-546, 2015.

3. Sadetzki S, Zach L, Chetrit A, Nass D, Hoffmann C, Ram Z, Zaaroor M, Umansky F, Rappaport ZH, Cohen A, et al: Epidemiology of gliomas in Israel: A nationwide study. Neuroepidemiology 31: 264-269, 2008.

4. Stupp R, Mason WP, van den Bent MJ, Weller M, Fisher B, Taphoorn MJ, Belanger $\mathrm{K}$, Brandes AA, Marosi C, Bogdahn U, et al: Radiotherapy plus concomitant and adjuvant temozolomide for glioblastoma. N Engl J Med 352: 987-996, 2005.

5. Lee JH, Jung TY, Jung S, Kim IY, Jang WY, Moon KS and Jeong EH: Performance status during and after radiotherapy plus concomitant and adjuvant temozolomide in elderly patients with glioblastoma multiforme. J Clin Neurosci 20: 503-508, 2013.

6. Liang BC, Thornton AF Jr, Sandler HM and Greenberg HS Malignant astrocytomas: Focal tumor recurrence after focal external beam radiation therapy. J Neurosurg 75: 559-563, 1991.

7. Héricé C, Khalil R, Moftah M, Boraud T, Guthrie M and Garenne A: Decision making under uncertainty in a spiking neural network model of the basal ganglia. J Integra Neurosci 15: 515-538, 2016

8. Zhu P, Wang Y, Wu J, Huang G, Liu B, Ye B, Du Y, Gao G, Tian Y, He L and Fan Z: LncBRM initiates YAP1 signalling activation to drive self-renewal of liver cancer stem cells. Nat Commun 7: 13608, 2016.

9. Liu B, Ye B, Yang L, Zhu X, Huang G, Zhu P, Du Y, Wu J, Qin X, Chen $\mathrm{R}$, et al: Long noncoding RNA $\operatorname{lncKdm} 2 \mathrm{~b}$ is required for ILC3 maintenance by initiation of Zfp292 expression. Nat Immunol 18: 499-508, 2017.

10. Smolle MA, Leithner A, Posch F, Szkandera J, Liegl-Atzwanger B and Pichler M: MicroRNAs in different histologies of soft tissue sarcoma: A comprehensive review. Int J Mol Sci 18: pii: E1960, 2017.

11. He L, He X, Lim LP, de Stanchina E, Xuan Z, Liang Y, Xue W, Zender L, Magnus J, Ridzon D, et al: A microRNA component of the p53 tumour suppressor network. Nature 447: 1130-1134, 2007.
12. Townley-Tilson WH, Callis TE and Wang D: MicroRNAs 1 , 133, and 206: Critical factors of skeletal and cardiac muscle development, function, and disease. Int J Biochem Cell Biol 42: $1252-1255,2010$

13. Zhang J, Xu D, Li N, Li Y, He Y, Hu X, Lyu L and He L: Downregulation of microRNA-31 inhibits proliferation and induces apoptosis by targeting HIF1AN in human keloid. Oncotarget 8: 74623-74634, 2017.

14. Zhou C, Jiang CQ, Zong Z, Lin JC and Lao LF: miR-146a promotes growth of osteosarcoma cells by targeting ZNRF3/ GSK-3 $\beta / \beta$-catenin signaling pathway. Oncotarget 8: 74276-74286, 2017.

15. Qu Y, Liu H, Lv X, Liu Y, Wang X, Zhang M, Zhang X, Li Y, Lou Q, Li S and Li H: MicroRNA-16-5p overexpression suppresses proliferation and invasion as well as triggers apoptosis by targeting VEGFA expression in breast carcinoma. Oncotarget 8: 72400-72410, 2017.

16. Zhou X, Wu W, Zeng A, Nie E, Jin X, Yu T, Zhi T, Jiang K, Wang Y,Zhang J and You Y: MicroRNA-141-3p promotes glioma cell growth and temozolomide resistance by directly targeting p53. Oncotarget 8: 71080-71094, 2017.

17. Zhao Y, Wang Y and Wang Y: Up-regulated miR-500a enhances hepatocarcinoma metastasis by repressing PTEN expression. Biosci Rep 37, 2017.

18. Degli Esposti D, Aushev VN, Lee E, Cros MP, Zhu J, Herceg Z, Chen $\mathrm{J}$ and Hernandez-Vargas H: miR-500a-5p regulates oxidative stress response genes in breast cancer and predicts cancer survival. Sci Rep 7: 15966, 2017.

19. Guo Y, Chen L, Sun C and Yu C: MicroRNA-500a promotes migration and invasion in hepatocellular carcinoma by activating the Wnt $/ \beta$-catenin signaling pathway. Biomed Pharmacother 91: 13-20, 2017.

20. Allen M, Bjerke M, Edlund H, Nelander S and Westermark B: Origin of the U87MG glioma cell line: Good news and bad news. Sci Transl Med 8: 354re3, 2016.

21. Livak KJ and Schmittgen TD: Analysis of relative gene expression data using real-time quantitative PCR and the 2(-Delta Delta C(T)) method. Methods 25: 402-408, 2001.

22. Gaur AB, Holbeck SL, Colburn NH and Israel MA: Downregulation of Pdcd 4 by mir-21 facilitates glioblastoma proliferation in vivo. Neuro Oncol 13: 580-590, 2011.

23. Xu YF, Hannafon BN, Zhao YD, Postier RG and Ding WQ: Plasma exosome miR-196a and miR-1246 are potential indicators of localized pancreatic cancer. Oncotarget 8: 77028-77040, 2017.

24. Rizzo S, Cangemi A, Galvano A, Fanale D, Buscemi S, Ciaccio M, Russo A, Castorina S and Bazan V: Analysis of miRNA expression profile induced by short term starvation in breast cancer cells treated with doxorubicin. Oncotarget 8: 71924-71932, 2017.

25. Li W, Yang W, Liu Y, Chen S, Chin S, Qi X, Zhao Y, Liu H, Wang J, Mei X, et al: MicroRNA-378 enhances inhibitory effect of curcumin on glioblastoma. Oncotarget 8: 73938-73946, 2017.

26. Zhu W, Qian J, Ma L, Ma P, Yang F and Shu Y: MiR-346 suppresses cell proliferation through SMYD3 dependent approach in hepatocellular carcinoma. Oncotarget 8: 65218-65229, 2017.

27. Liu N, Zhang L, Wang Z, Cheng Y, Zhang P, Wang X, Wen W, Yang H, Liu H, Jin W, et al: MicroRNA-101 inhibits proliferation, migration and invasion of human glioblastoma by targeting SOX9. Oncotarget 8: 19244-19254, 2017.

28. Jiang C, Long J, Liu B, Xu M, Wang W, Xie X, Wang X and Kuang M: miR-500a-3p promotes cancer stem cells properties via STAT3 pathway in human hepatocellular carcinoma. J Exp Clin Cancer Res 36: 99, 2017.

29. Mei Z, He Y, Feng J, Shi J, Du Y, Qian L, Huang Q and Jie Z: MicroRNA-141 promotes the proliferation of non-small cell lung cancer cells by regulating expression of PHLPP1 and PHLPP2. FEBS Lett 588: 3055-3061, 2014.

30. Baykara O, Tansarikaya M, Bulut P, Demirkaya A and Buyru N: CHD5 is a potential tumor suppressor in non small cell lung cancer (NSCLC). Gene 618: 65-68, 2017.

31. Wang L, He S, Tu Y, Ji P, Zong J, Zhang J, Feng F, Zhao J, Gao G and Zhang Y: Downregulation of chromatin remodeling factor CHD5 is associated with a poor prognosis in human glioma. J Clin Neurosci 20: 958-963, 2013.

This work is licensed under a Creative Commons Attribution-NonCommercial-NoDerivatives 4.0 International (CC BY-NC-ND 4.0) License. 